\title{
Evaluating Throughput and Delay in 3G and 4G Mobile Architectures
}

\author{
Eralda Caushaj', Ivan Ivanov', Huirong Fu'2, Ishwar Sethi' ${ }^{2}$, Ye Zhu ${ }^{3}$ \\ ${ }^{1}$ Empire State College, State University of New York, New York, USA \\ ${ }^{2}$ Oakland University, Rochester Hills, USA \\ ${ }^{3}$ Cleveland State University, Cleveland, USA \\ Email: eralda.caushaj@esc.edu, ivan.ivanov@esc.edu, fu@oakland.edu, isethi@oakland.edu, \\ y.zhu61@csuohio.edu
}

Received 14 May 2014; revised 16 June 2014; accepted 16 July 2014

Copyright (C) 2014 by authors and Scientific Research Publishing Inc.

This work is licensed under the Creative Commons Attribution International License (CC BY).

http://creativecommons.org/licenses/by/4.0/

(c) †

\begin{abstract}
All mobile carriers these days have provided 3G and 4G services to their customers. The evolution of cellular networks from $3 \mathrm{G}$ to $4 \mathrm{G}$ has improved several performance metrics of the data communications. The focus of this paper is to evaluate two of the most important performance metrics: throughput and delay. We consider the cellular network as an integrate infrastructure that includes mobile and fixed nodes. Based on this model we calculate and analyze the throughput and delay. Our results illustrate that the throughput is increased while the delay is decreased in 4G data network compared to the previous $3 G$ architecture. In addition, we evaluate how the delay affects the security of the network.
\end{abstract}

\section{Keywords}

Network, Throughput, Delay, Architecture, Graph

\section{Introduction}

The third generation (3G) system was officially completed in 1997 by the International Telecommunications Union Radio communication Sector (ITU-R). This technology was a leader for more than a decade in the cellular network architecture. When 4G was introduced as an upgrade of the existing architecture, it was driven by the increasing demand for mobile broadband services with higher data rates and Quality of Service (QoS) [1]. The 4G infrastructure market in 2014 is predicted to reach $\$ 11.4$ billion. According to AT \& T the number of 4G subscribers will reach 440 million [2].

The method introduced by Gupta and Kumar calculates the optimal delay on throughput for a fixed random 
network [3]. The nodes of cellular network can be classified as fixed and mobile; therefore this method we will apply for some nodes of cellular network characterizing the immobile network. Another method introduced by four researchers at Stanford University calculates the throughput and delay for mobile nodes [4]. They take in consideration the velocity of the nodes, average distance traveled in one hop and the number of nodes involved in the network.

The main contribution in this paper is the approach to evaluate two performance metrics such as throughput and delay in 3G/4G network by proposing a new model that consists as a combination of two existing previously described methods. The implications of delay in both architectures are explained as well.

In this paper, based on our research and analysis, we intend to shed more light on this topic. In Section 2 we describe the architecture of a mobile cellular network, by detailing all components of the 3G/4G network. We define in Section 3 the new model by classifying all the nodes of the architecture in mobile and fixed ones and calculate throughput and delay for both architectures. Finally, Section 4 presents the conclusion of this research and directions for future work.

\section{Mobile Cellular Architecture}

The enormous success of wireless mobile communications is based on the rapid pace of hardware miniaturization and technology innovations. From the 3G architecture first launched in 1997, the wireless mobile network has been transformed to a network that can transport rich multimedia contents. Moreover, the 4G wireless systems are designed to use IP for all services.

\subsection{G Data Network}

Figure 1 shows the fundamental architecture of a wireless cellular network [5]. The base stations (BS) and the base station controllers (BSC) are the key components of a wireless cellular network (WCN).They perform all functions necessary to maintain radio connections to a cell phone, by coding/decoding the antennae voice [6]. Each BS comprises all radio equipment, i.e. antennas, signal processing, amplifiers necessary for radio transmission. The BSC reserves radio frequencies, handles the handover from one BS to another within the BSS. Also it is responsible for performing paging on the Smartphone. The mobile switching center (MSC), serving general packet radio service support nodes (SGSN) and gateway (GPRS) support nodes (GGSN) are connecting the wireless network with a public switch telephone network (PSTN) and the internet. The MSC performs submissions between different BSCs and also supports charging, accounting, and roaming of users between different providers in different countries [7]. The network switching systems consists of a home location register (HLR), visitor location registers (VLR), and mobile switching centers (MSC). The HLR is the most important database in the system, because it stores all user-relevant information, i.e. static information, such as subscribed services, and dynamic information, such as the user location. HLR can manage data for several million customers [8]. The VLR (Visitor Location Registers) are dynamic databases which store all important information for mobile users in the location area they control. Each VLR is associated with an MSC which handles all signaling needed

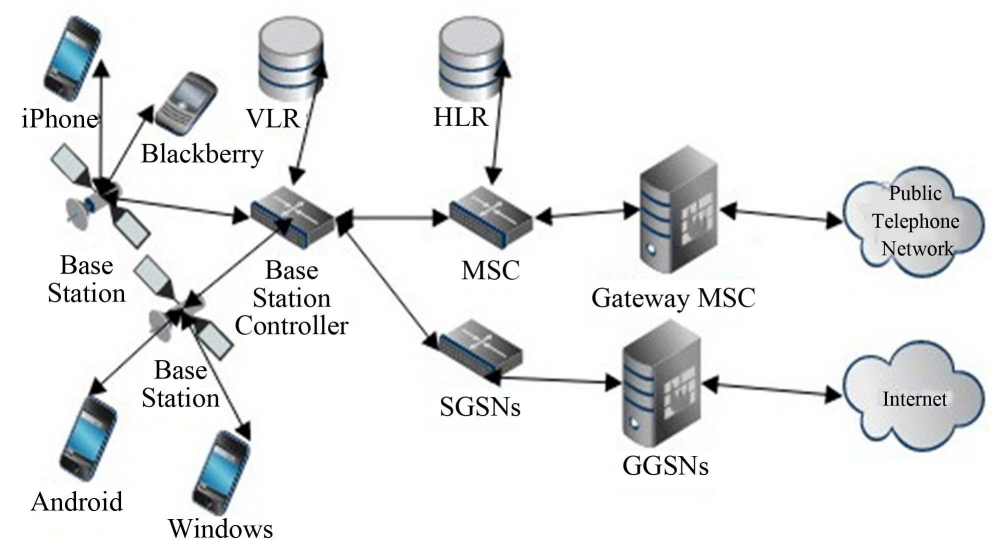

Figure 1. Centralized Wireless Cellular Network (WCN) architecture and its components for voice and data transmission [2]. 
for connection setup, connection release and handover to another MSC. All functions needed for supplementary services, such as call forwarding, multi-party calls, and reverse charging are performed by every MSC. There are two types of nodes in the 3G core network: Serving GPRS Support Nodes (SGSN) and Gateway GPRS Support Nodes (GGSN). The SGSNs are responsible for delivering datagram to/from the mobile nodes in the radio access networks to which the SGSN are attached. The SGSNs interact with MSC to provide user authorization and handoff and act as a gateway connecting many SGSN. The Internet is necessary to access the web and database servers. Mobile switching center is the component associated with call set-up, release, and routing in WCN. VLR is a database in an ongoing process of updating records about the subscribers roaming within a MSC location area. Home Location Register is the permanent database that contains information regarding subscribers authorized to use a GSM network. Subscriber Identity Module (SIM) serves as the primary key for each HLR record. Serving GPRS support nodes route packets to and from the geographical SGSN area. SGSN stores location information and user profiles of all GPRS users registered with this SGSN. Gateway GPRS Support Nodes interface with external IP packet networks. Each VLR is associated with an MSC which handles all signaling needed for connection setup, connection release and handover to other MSC. All functions are needed for supplementary services, such as call forwarding, multi-party calls, and reverse charging are performed by every MSC. There are two types of nodes in the 3G core network: Serving GPRS Support Nodes (SGSN) and Gateway GPRS Support Nodes (GGSN) the SGSN responsible for delivering datagram to/from the mobile nodes in the radio access networks to which the SGSN is attached. The SGSN interact with MSC providing user authorization and handoff, and act as gateway connecting many SGSN. Internet is necessary to access the web and database servers.

\subsection{G Data Network}

Long Term Evolution-Advanced (LTE-Advanced) and System Architecture Evolution (SAE) are unified as 4G wireless network.SAE is an upgrade of the GPRS Core Network, with the following differences:

- Simplified architecture, fewer components are involved in the data network compared to 3G architecture.

- Support for higher throughput, we are going to evaluate it in Section 3.

- Support for mobility between, multiple heterogeneous access networks.

As carriers all over the US have millions of subscribers for both 3G and4G architectures, we present in Figure 2 an integrated network diagram [1] [2]. The carriers' networks are divided in three key segments:

- Radio Access Network (RAN)provides wide-area wireless connectivity to mobile devices and is considered as a collection of base stations. The base station (eNodeB) schedules all the transmissions that the mobile carries out on the physical uplink shared channel [9] [10].

- Evolved Packet Core (EPC) is a flat all-IP-based considered as the core of SAE. The EPC is the equivalent of GPRS networks and includes Mobility Management Entity (MME), Serving Gateway (S-GW) and Packet Data Network Gateway (PDN-GW) [1] [2].

- MME main role is to manage security functions such as authentication, authorization, and Non-Access Stratum (NAS) signaling. Also it is responsible for handling idle state mobility, roaming, and handovers [1] [2].

Serving Gateway (S-GW) is connected to a UE and handles several functions such as packet routing and forwarding [1] [2].

PDN-GW assigns an IP address from the PDN to UE and provides access to it [1] [2].

IMS network intends to provide circuit-switched network subscribers with services built and based on the Internet applications, services and protocols. The intention of IMS network provides convergence and access to various services such as data, voice, messaging, video and web related technologies to both fixed-line subscribers and wireless-users [11].

Home Subscriber Server (HSS) is a central database that contains user and subscription-related information. Thereare several functions that HSS handles such as mobility management, call and session establishment support, user authentication and access authorization [11].

LTE provides 2 to 5 time greater spectral efficiency than most advanced 3G networks, and a lower cost per bit. Also the 4G network offers faster downloads up to $100 \mathrm{Mbit} / \mathrm{s}$ initially and a better user experience [2].

\section{New Model to Calculate Throughput and Delay in Data Network}

Two of the most important performance metrics of a data network are throughput and total nodal delay required 


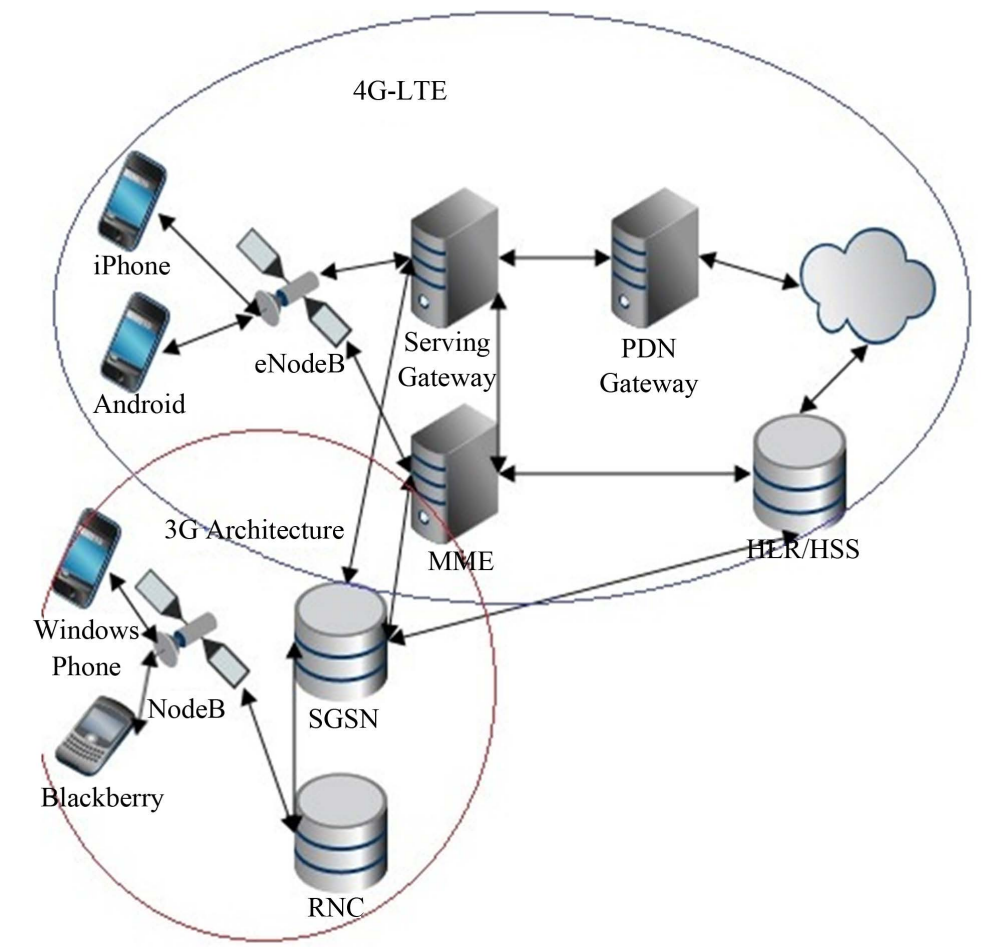

Figure 2. 3G-4G architecture and its components for voice and data transmission [1].

to deliver a packet from origin to destination. The total nodal delay is calculated as the sum of processing, queuing, transmission and propagation delay [7].

1) The processing delay represent the time between when the packet is correctly received at the head node of the link and the time the packet is assigned to an outgoing link queue for transmission.

2) The queuing delay between the time the packet is assigned to a queue for transmission and the time it starts being transmitted.

3) The transmission delay between represent the time required that the first and last bits of the packet are transmitted.

4) The propagation delay between the time the last bit is transmitted at the head node of the link and the time the last bit is received at the tail node.

Researchers at Stanford University define $T(n)$ as the maximum delay-constrained throughput and when there is no delay, $T(n)$ is the throughput capacity. The number of nodes involved in the network is given by the parameter $n$ [3]. The definition of $\theta$-function is that for every function $f(n)=\theta(f(n))$ where $f(n)=O(g(n)) ; g(n)=O(f(n))$. Gupta and Kumar introduced a random network model for calculating the throughput and delay in a wireless network when the nodes are fixed [3] and researchers at Stanford University calculated throughput and delay in a mobile network considering node mobility and queueing [2]. These models do not take queueing delay at the source into account, since the interest is in the network delay. $D(n)$ represents the delay of a packet in a network and is the time it takes the packet to reach the destination after it leaves the source.

Our goal is to calculate the throughput and delay for both network architectures-3G and 4G, based on the previously suggested calculation models. We mapped each network architecture in bi-directional graphs and considered the smartphone and wireless base stations as mobile nodes and the rest of architecture as fixed wireless network. Therefore the throughput and delay is combination of the earlier models with different number of nodes involved.

\subsection{Throughput and Delay 3G Architecture}

The following diagram, Figure 3 represents the 3G network as a bi-directional graph when each node is an im- 


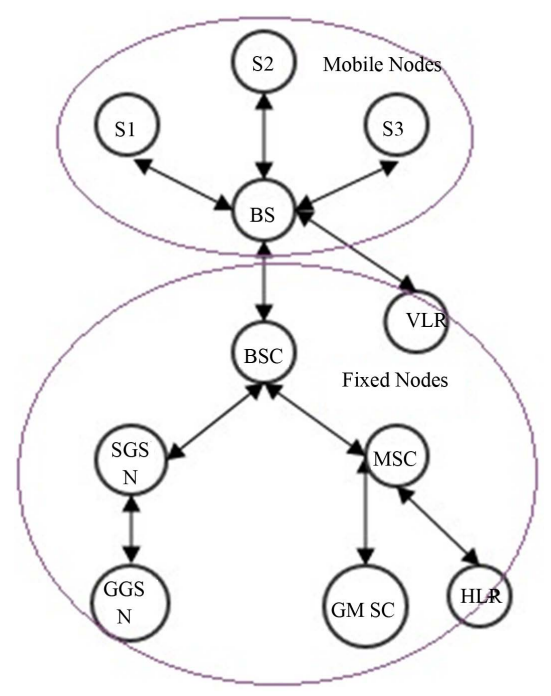

Figure 3. 3G bi-directional graph.

portant component of the architecture. The nodes are classified as mobile and fixed nodes. In the first category we have smartphones and the base stations. Base station controllers, mobile switching centers, serving general packet radio service support nodes, gateway (GPRS) support nodes, home location register, visitor location register and gateway mobile switching centers are all fixed nodes in the architecture. All the nodes in the network serve as sender/receivers.

Throughput and Delay in Fixed wired network:

$$
\begin{aligned}
& D(n)=\theta(n * T(n)), \text { for } T(n)=\theta(1 / \sqrt{n * \log (n)}) \\
& \text { and } D(n)=\theta(\sqrt{n} / \sqrt{\log n})
\end{aligned}
$$

There are seven fixed nodes in 3G architecture that are considered as part of the fixed wired network. Therefore the throughput and delay are calculated as follows:

$$
\begin{aligned}
& T(7)=O(1 / \sqrt{7 * \log (7)})=\theta(0.41) \\
& D(7)=\theta(\sqrt{7} / \sqrt{\log 7})=\theta(2.88)
\end{aligned}
$$

The two nodes out of $3 \mathrm{G}$ architecture that are considered as part of the mobile network are the smartphone device and base station.

Throughput and Delay in a mobile network:

$$
\begin{aligned}
& T(n)=\theta(1 / \sqrt{n * a(n) * \log n}) \text {, and } D(n)=\theta(1 / v(n) * \sqrt{a(n)}) \\
& \text { when } \theta(\log n / n)<a(n)<\theta(1) \text { and } v(n)=\theta(1 / \sqrt{n})
\end{aligned}
$$

The trade-off is parameterized by $a(n)$, where $\sqrt{a(n)}$ corresponds to the average distance traveled in one hopand $v(n)$ the velocity of the node.

Throughput for $3 \mathrm{G}$ data network:

$$
\begin{gathered}
T(2)=\theta(1 / \sqrt{2 * a(2) * \log 2}), \text { where } \\
\theta(\log 2 / 2)<a(2)<\varsigma(1) \\
\theta(0.15)<a(2)<\theta(1) \text { and } \\
\theta(1 / 0.77 * \sqrt{\theta(1)})<T(2)<\theta(1 / 0.77 * \sqrt{\theta(0.15)})
\end{gathered}
$$

Delay for 3G data network: 


$$
\begin{aligned}
& D(2)=\theta(1 /(v(2) * \sqrt{(a(2))}))=\theta(1 / \theta(1 / \sqrt{2}) * \sqrt{(a(2))}) \\
& \text { when } \theta(0.15)<a(2)<\theta(1) \\
& \theta(1 / \theta(0.7) * \sqrt{(\theta(1))})<D(2)<\theta(1 / \theta(0.7) * \sqrt{(\theta(0.15))})
\end{aligned}
$$

Total Throughput for 3G data network:

$$
\begin{aligned}
\theta(0.41)+\theta(1 / 0.77 * \sqrt{\theta(1)}) & <T(9)=T(7)+T(2) \\
& <\theta(0.41)+\theta(1 / 0.77 * \sqrt{\theta(0.15)})
\end{aligned}
$$

Total Delay for 3G data network:

$$
\begin{aligned}
\theta(2.88)+\theta(1 / 0.77 * \sqrt{\theta(1)}) & <D(9)=D(7)+D(2) \\
& <\theta(2.88)+\theta(1 / 0.77 * \sqrt{\theta(0.15)})
\end{aligned}
$$

\subsection{Throughput and Delay 4G Architecture}

The following diagram, Figure 4 represents the 4G network as a bi-directional graph when each node is an important component of the architecture. The nodes are classified as mobile and fixed nodes. In the first category we have smartphones and the eNodeB. Actually the 4G bi-directional graph is very similar to the 3G graph with most important additional direct link between SG and MME nodes in the fixed network component which increases reliability and flexibility of communications.

Throughput and Delay in Fixed wired network:

Based on Equation (1) we can calculate throughput and delay in fixed wired network for 4G architecture. There are four fixed nodes in $4 \mathrm{G}$ architecture that are considered as part of the fixed wired network. Therefore the throughput and delay are calculated as follows:

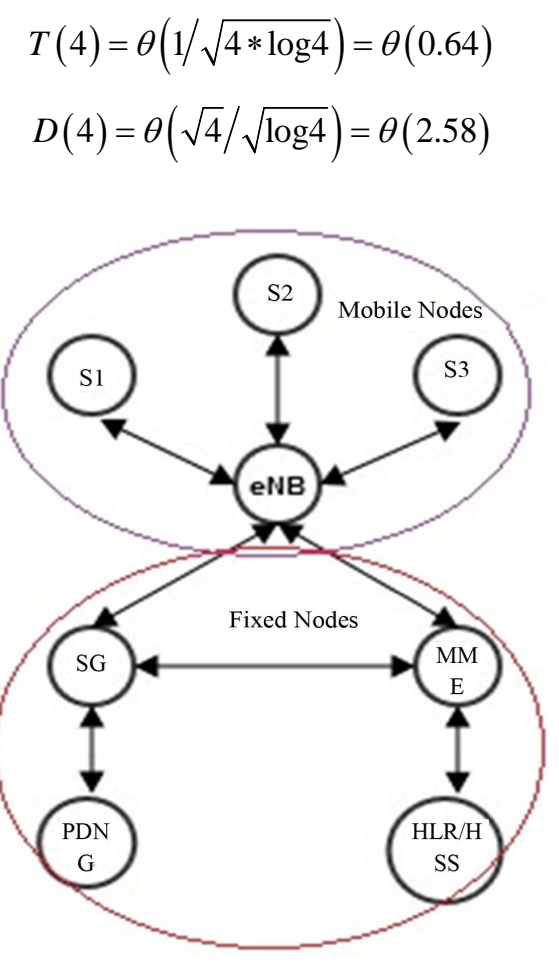

Figure 4. 4G bi-directional graph. 
Throughput and Delay in a mobile network:

There are two nodes out of 3G architecture that are considered as part of the mobile network, the smartphone device and base station.

Based on Equation (2) we can calculate the throughput and delay for 4G data network as follows:

Throughput for $4 \mathrm{G}$ data network:

As the number of mobile nodes in both architectures is the same, therefore the throughput and delay for $4 \mathrm{G}$ network will be the same as for $3 \mathrm{G}$ architecture.

$$
\begin{gathered}
T(2)=\theta(1 / \sqrt{2 * a(2) * \log 2}), \text { where } \\
\theta(\log 2 / 2)<a(2)<\theta(1) \\
\theta(0.15)<a(2)<\theta(1) \text { and } \\
\theta(1 / 0.77 * \sqrt{\theta(1)})<T(2)<\theta(1 / 0.77 * \sqrt{\theta(0.15)})
\end{gathered}
$$

Delay for 4G data network:

$$
\begin{aligned}
& D(2)=\theta(1 /(v(2) * \sqrt{(a(2))}))=\theta(1 / \theta(1 / \sqrt{2}) * \sqrt{(a(2))}) \\
& \text { when } \theta(0.15)<a(2)<\theta(1) \\
& \theta(1 / \theta(0.7) * \sqrt{(\theta(1))})<D(2)<\theta(1 / \theta(0.7) * \sqrt{(\theta(0.15))})
\end{aligned}
$$

Total Throughput for 4G data network:

$$
\begin{aligned}
\theta(0.64)+\theta(1 / 0.77 * \sqrt{\theta(1)}) & <T(6)=T(4)+T(2) \\
& <\theta(0.64)+\theta(1 / 0.77 * \sqrt{\theta(0.15)})
\end{aligned}
$$

Total Delay for 4G data network:

$$
\begin{aligned}
\theta(2.58)+\theta(1 / 0.7 * \sqrt{\theta(1)}) & <D(6)=D(4)+D(2) \\
& <\theta(2.58)+\theta(1 / 0.7 * \sqrt{\theta(0.15)})
\end{aligned}
$$

\section{Conclusions and Future Work}

The focuses of our analysis and calculations are two performance metrics such as throughput and delay in both architectures. Regarding the delay we concentrate on average delay without considering the queue delay. The cellular network has two different kinds of nodes, mobile and fixed. In both architectures the number of mobile nodes is the same two: a smartphone and base station or eNB for 4G architecture, therefore the throughput and the delay for both architectures is the same as represented in Table 1. For the delay there are three parameters considered for each node such as average distance traveled in one hop, velocity of the node, and the number of hops [4]. The difference of both parameters will be noticed in the fixed node architecture when the number of nodes is different. The more number of nodes involved in the architecture the more is the delay in communica-

Table 1. Summary of total throughput and delay for 3G/4G network data.

\begin{tabular}{ccc}
\hline \multirow{2}{*}{ Network Architecture } & Throughput & Performance Metrics \\
\cline { 2 - 3 } 3G (fixed node) & $\theta(0.41)$ & $\theta(2.88)$ \\
4G (LTE) (fixed node) & $\theta(0.64)$ & $\theta(2.58)$ \\
Mobile node & {$[\theta(1 / 0.77 * \sqrt{\theta(1)}), \theta(1 / 0.77 * \sqrt{\theta(0.15)})]$} & {$[\theta(1 / \theta(0.7) * \sqrt{\theta(1)}, \theta(1 / \theta(0.7) * \sqrt{\theta(0.15)}))]$} \\
\hline
\end{tabular}


tion and the throughput is less. The model doesn't consider the transmission rate of both architectures even though it is clear that in $4 \mathrm{G}$ architecture is considerably higher. We were able to show that the throughput for $4 \mathrm{G}$ just for the fixed nodes is $\theta(0.64)$ compared to $\theta(0.41)$ in 3G. The delay is smaller in $4 \mathrm{G}(2.58)$ compared to $\theta(2.88)$ in 3G. The most important parameters in both calculations are the number of nodes in each architecture. The fewer components involved in an architecture, the better is the overall performance of the data network. The delay as a performance metric of the network is crucial when it comes to the overall security of the architecture, therefore the smaller the delay less security risks are evident to the network. The KISS design rule (Keep it Simple Stupid) in security should be applied when it comes to network architectures as well.

As the current trends in mobile communications involve additional either third party or vendor specific mobile security management systems, our future research will be focused on how those solutions alternate the current 3G/4G architectures and we will extend our performance metrics approach to run series of quantitative analyses on different architectural options.

\section{Acknowledgements}

This material is based upon work supported by the National Science Foundation under Grant No. 1062960. Any opinions, findings, and conclusions or recommendations expressed in this material are those of the author(s) and do not necessarily reflect the views of the National Science Foundation.

\section{References}

[1] Akyildiz, I., Gutierrez-Estevez, D.M. and Reyes, E. (2010) The Evolution to 4G Cellular Systems: LTE-Advanced. Physical Communication, 3, 217-244. http://dx.doi.org/10.1016/j.phycom.2010.08.001

[2] Stanley, S. (2010) LTE Technology and Components. http://www.iphase.com/downloads/LTE Technology and Components 2010 LRWebinar.pdf

[3] Gupta, P. and Kumar, P.R. (2000) The Capacity of Wireless Networks. IEEE Transactions on Information Theory, 46, 388-404. http://dx.doi.org/10.1109/18.825799

[4] Gamal, A., Mammen, J., Prabhakar, B. and Shah, D. (2204) Throughput-Delay Trade-Off in Wireless Networks. INFOCOM, Twenty-Third Annual Joint Conference of the IEEE Computer and Communications Societies, 475-485.

[5] Venkataram, P. and Satish, B. (2010) Wireless and Mobile Network Security. Tata McGraw-Hill Education, New Dehli.

[6] Caushaj, E., Fu, H., Sethi, I., Badih, H., Watson, D., Zhu, Y. and Leng, S. (2013) Theoretical Analysis and Experimental Study: Monitoring Data Privacy in Smartphone Communications. IJITN.

[7] Kurose, J. and Ross, K. (2012) Computer Networking: A Top-Down Approach. Pearson, 6th Edition.

[8] Beaubrun, R., Moulin, B. and Jabeur, N. (2007) Architecture for Delivering Location-Based Services. IJCSNS International Journal of Computer Science and Network Security, 7.

[9] Gudipati, A., Perry, D., Erran, L. and Katti, S. (2013) SoftRAN: Software Defined Radio Access Network. HotSDN 2013 Proceedings of the 2nd ACM SIGCOMM Workshop on Hot Topics in Software Defined Networking, 25-30.

[10] Cox, C. (2012) An Introduction to LTE: LTE, LTE-Advanced, SAE and 4G Mobile Communications. Wiley, 151. http://dx.doi.org/10.1002/9781119942825.ch9

[11] Bhadrapur, P.K. (2012) HSS Front-End Implementation for a Large Scale Common HLR/HSS. http://uu.diva-portal.org/smash/get/diva2:559134/FULLTEXT01.pdf 
Scientific Research Publishing (SCIRP) is one of the largest Open Access journal publishers. It is currently publishing more than 200 open access, online, peer-reviewed journals covering a wide range of academic disciplines. SCIRP serves the worldwide academic communities and contributes to the progress and application of science with its publication.

Other selected journals from SCIRP are listed as below. Submit your manuscript to us via either submit@scirp.org or Online Submission Portal.
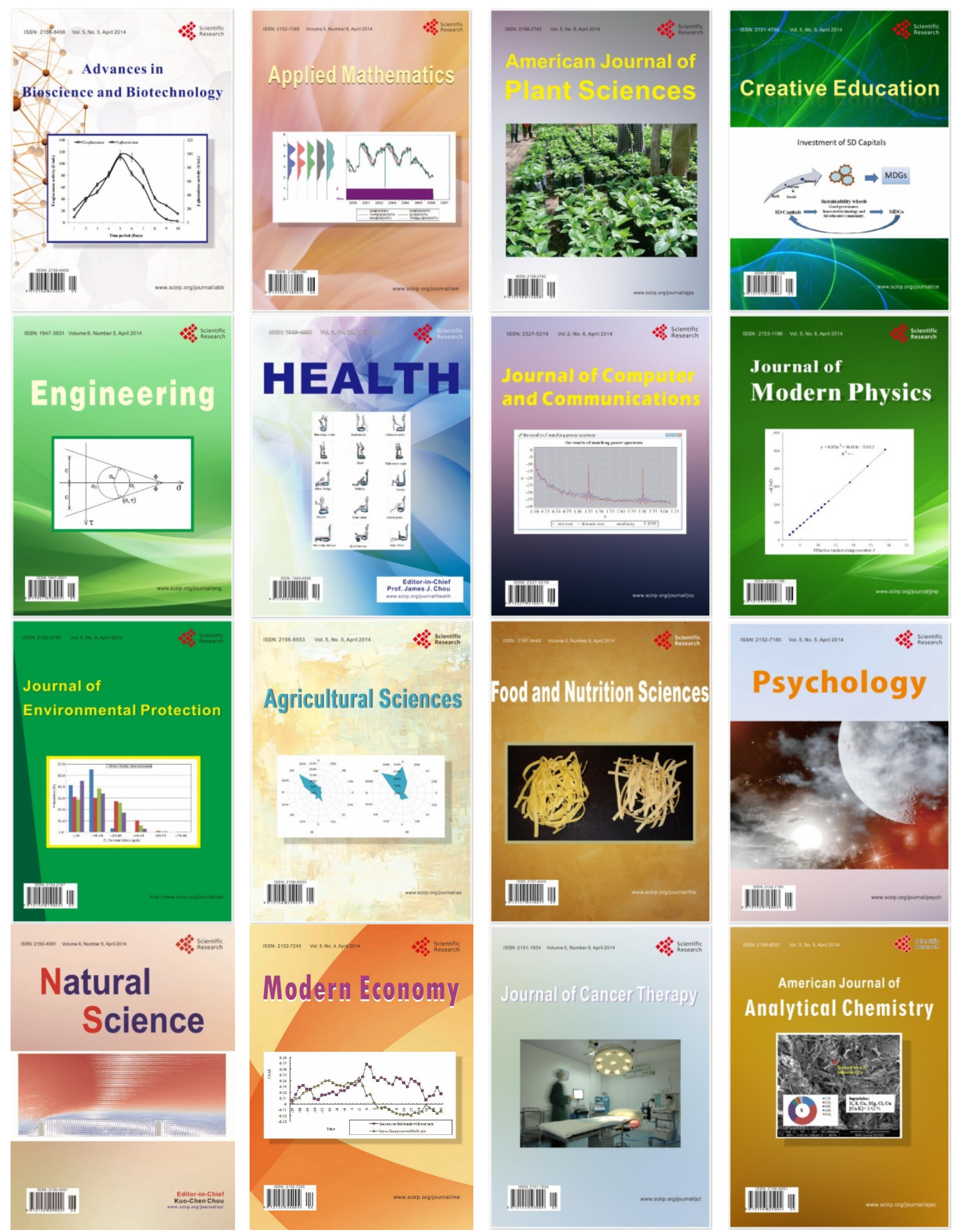